\title{
Análisis Comparativo de la Composición Corporal de Árbitros de Fútbol de Brasil y Uruguay
}

\author{
Comparative Analysis of Body Composition of Football (Soccer) Referees \\ from Brazil and Uruguay
}

*Alberto Inácio da Silva; ${ }^{* *}$ Héctor de los Santos \& ${ }^{* * *}$ Carlos Cabrera

DA SILVA, A. I.; DE LOS SANTOS, H. \& CABRERA, C. Análisis comparativo de la composición corporal de árbitros de fútbol de Brasil y Uruguay. Int. J. Morphol., 30(3):877-882, 2012.

RESUMEN: El objetivo de este estudio fue hacer un análisis comparativo del perfil morfológico de árbitros de fútbol de elite de Brasil y Uruguay. La muestra fue compuesta por 27 árbitros del Brasil, con edad promedio de 37,6 $\pm 4,2$ años, peso de 79,1 $\pm 7,9 \mathrm{~kg}$ y estatura de $179,1 \pm 4,8 \mathrm{~cm}$ y 14 árbitros de Uruguay con edad promedio de $37,8 \pm 3$ años, peso de $74,3 \pm 7,4 \mathrm{~kg}$ y estatura de $175 \pm 6,8 \mathrm{~cm}$. Las variables de masa corporal, estatura, grosor de pliegues cutáneos, circunferencias corporales y diámetros óseos, fueron medidas con la finalidad de estimar la composición corporal y determinar el somatotipo de los árbitros. Para determinar la composición corporal se utilizaron: siete pliegues cutáneos, siete circunferencias corporales y cuatro diámetros óseos. Con respecto a los datos de composición corporal cuando se comparó los datos de los árbitros brasileños con los uruguayos se verificó una diferencia estadísticamente significativa entre el peso corporal, IMC y porcentaje de grasa corporal ( $p>0,05)$. Aunque el somatotipo de ambos grupos serán clasificados como endomorfo-mesomorfo (somatotipo promedio de 3,7-4,0 -2,0), los árbitros brasileños presentaron una porcentaje de grasa corporal muy elevada lo que puede ser un factor limitante en la performance física durante el partido y que podría ser controlado por medio de programas de buenos hábitos alimenticios como de acondicionamiento físico.

PALABRAS CLAVES: Árbitros; Fútbol; Composición corporal.

\section{INTRODUCIÓN}

El fútbol es uno de los deportes más practicados en el mundo (Fuke et al., 2009). Constituye uno de los fenómenos sociales más estridente en los tiempos modernos, moviliza a miles de personas directa e indirectamente, haciendo de ésto uno de los segmentos más rentables en términos de marketing, publicidad y comercialización. Una encuesta oficial llamada "Big Count 2006" llevada a cabo por la Fédération Internationale de Football Association (FIFA) ha puesto de manifiesto que el año 2006 estaban registrados en el mundo más de 840.000 árbitros y árbitros asistentes (Bizzini et al., 2009). Debido a su importancia para el fútbol el equipo de arbitraje ha sido objeto de numerosos estudios con diferentes enfoques (Reilly \& Gregson, 2006).

Los grandes avances en el fútbol direccionados a la preparación física fueron impulsados por los avances en la metodología del entrenamiento deportivo y la fisiología del ejercicio. Sin embargo, una parte importante de ellos fue generado por el regreso de los Juegos Olímpicos (Dantas,
2003). Desde la creación oficial del fútbol en Inglaterra en 1863, el desarrollo de la aptitud física de los jugadores se volvió más especializado, así como la intensidad de las acciones motoras de ellos aumenta cada día. Este aumento en la dinámica del juego modificó la posición de la FIFA y pasó a exigir un mayor nivel de aptitud física de los árbitros y desarrollar una batería de pruebas físicas para evaluarlos (Rontoyannis et al., 1998).

Una de las primeras medidas tomada por la FIFA para evaluar sus árbitros ocurrió en 1994, en dicha ocasión todos los árbitros que trabajaron durante la Copa del Mundial de 1994, pasaron por el “The Cooper Institute”, en Dallas. Además, a partir de esa fecha los árbitros pasaron a ser evaluados periódicamente. La primera batería de tests físicos desarrollada por la FIFA era compuesta por : una carrera de 12 minutos (test de Cooper), dos carreras de 50 metros, dos carreras de 200 metros y un test de agilidad (4 x $10 \mathrm{~m})$, prueba que fue abolida en 1995 (Rontoyannis et al.).

* Docente da Universidade Estadual de Ponta Grossa - Paraná - Brasil.

** Posgrado Medicina del Deporte. Facultad de Medicina. UDELAR, Uruguay.

**** Posgrado Medicina del Deporte. Facultad de Medicina. UDELAR, Uruguay. Arbitro Asistente / Asociación Uruguaya de Fútbol/ Colegio de Árbitros. 
Sin embargo, datos globales muestran un aumento en el predominio de sobrepeso y obesidad en la población, que varía de acuerdo con la edad, sexo, raza y clases socioeconómicas de cada región (Bouchard, 2003). En las evaluación de los atletas y detección del acondicionamiento actual, determinadas características físicas y morfológicas son analizadas. Con relación a las características morfológicas evaluadas, la cuantificación de los componentes corporales y principalmente entre éstos el porcentaje de grasa $(\% \mathrm{G})$ y de masa muscular $(\% \mathrm{MM})$, son valores preferentemente observados por los evaluadores (Guedes \& Guedes, 2006).

De acuerdo con Cuchiaro (2000), para establecer programas específicos de entrenamiento es necesario conocer el perfil antropométrico del atleta, para saber si éste se encuentra alejado del perfil adecuado para la función, además de las exigencias físicas de la actividad. Se sabe también que cantidades elevadas de grasa corporal perjudican el desempeño de los individuos, además de constituir un factor de riesgo de diversas enfermedades. Por lo tanto, es fundamental el control adecuado de la adiposidad corporal. Así, la Confederación Brasileña de Fútbol (CBF), impuso en sus exigencias para la evaluación física de sus árbitros el 2011 que se mida el porcentaje de grasa corporal. Considerando que estos datos van a influenciar en la calificación del árbitro para actuar en juego nacionales.

Frente de esto, se plantea la necesidad de realizar estudios que aborden el perfil morfológico de los árbitros de fútbol. Por lo tanto, el objetivo de este trabajo fue evaluar y comparar el perfil morfológico de los árbitros de élite de Brasil y Uruguay, dos países que tienen tradición en el fútbol mundial.

\section{METODOLOGÍA}

La muestra fue compuesta por 27 árbitros brasileños pertenecientes a la Federación Paranaense de Fútbol (FPF) registrados por la Confederación Brasileña de Fútbol (CBF) y 14 árbitros uruguayos del Colegio de Árbitros, dependiente de la Asociación Uruguaya de Fútbol (A.U.F), todos de sexo masculino. La evaluación de los árbitros brasileños fue realizada en la ciudad de Curitiba en Brasil mientras que los uruguayos fue hecha en la ciudad de Montevideo, Uruguay.

Los procedimientos adoptados en este estudio siguen la Resolución 196/96, del Consejo Nacional de Salud de Brasil, que trata de los procedimientos de pesquisa en seres humanos. Fue autorizado el estudio por la dirección del Colegio de Árbitros y su departamento Físico. Se siguieron las normas éticas de la Asociación Médica Mundial.
El proyecto fue aprobado por la Comisión de Ética en Pesquisa de la Universidad Estadual de Maringá - UEM (Parecer 601/2010). Antes del inicio de la recolección de los datos, fueron informados los propósitos y procedimientos técnicos que serian adoptados, enfocando los objetivos y beneficios de la investigación para los participantes, a través de una reunión del responsable de la investigación, junto con el preparador físico de los árbitros, así como de toda la muestra de estudio. En esta reunión fue garantizada la libertad de asistencia de participación en el estudio por parte de los árbitros antes y durante las evaluaciones.

Para la evaluación de la composición corporal fueron medidos nueve pliegues cutáneos (subescapular, tríceps, bíceps, pectoral, axilar promedio, abdomen, suprailíaca, muslo y pantorrilla) utilizando un adipómetro marca Cescorf de fabricación brasileña, con una precisión de medida de $0,1 \mathrm{~mm}$. También se midieron nueve circunferencias corporales (antebrazo, brazo contraído, brazo relajado, tórax, abdomen, cadera, muslo superior, muslo mediano y pantorrilla) utilizando una cinta métrica flexible marca Cescorf, con una graduación de $0,1 \mathrm{~cm}$. A su vez, se realizó la medición de cuatro diámetros óseos (biestiloide, biepicondiliano del húmero, biepicondiliano del fémur y bimaleolar), empleando un antropómetro de metal o calibre óseo, marca Mitutoyo, con una precisión de $0,1 \mathrm{~mm}$. A la evaluación de estas variables antropométricas (pliegues cutáneos, circunferencias corporales y diámetros óseos), siguieron las estandarizaciones propuestas por Lohman et al. (1991).

Se determinó además la masa corporal, la estatura y la edad conforme Gordon et al. (1991). La densidad corporal fue determinada a partir de las variables antropométricas utilizando la ecuación propuesta por Jakson \& Pollock (1978), que utiliza la suma de 7 pliegues cutáneos y dos perímetros y el porcentual de gordura por la ecuación de Siri (1961).

Para fines de comparación, los somatotipos fueron clasificados en categorías de acuerdo con Carter (2002). El somatotipo también fue expuesto en un gráfico (somatocarta), desarrollada por Carter \& Heath (1990), donde fueron calculados los valores de las coordenadas X y Y: $\mathrm{X}=$ ectomorfia - endomorfia; $\mathrm{Y}=2 \mathrm{x}$ mesomorfia (endomorfia + ectomorfia).

Los resultados son expresados como valores promedios con su desviación estándar. Los valores promedios fueron comparados por el test " $t$ " de Student. Los resultados fueron considerados estadísticamente significativos cuando la probabilidad de la ocurrencia de hipótesis nula fuera menor que 0,05 . La diferencia entre el somatotipo de los dos grupos de árbitros también se analizó por medio de la dis- 
tancia de dispersión del somatotipo (DDS) (Hebbelinck et al., 1975). DDS permite verificar la distancia entre dos somatotipos, estableciendo que la distancia es estadísticamente significativa cuando la DDS es 2 .

\section{RESULTADOS}

En la Tabla I son presentadas las características antropométricas de los árbitros brasileños y uruguayos. Mientras los valores del IMC de ambos grupos están dentro del padrón de normalidad, pero una investigación más detallada de los datos referentes al IMC permitió detectar que apenas el $56 \%$ de los árbitros brasileños están con el IMC dentro del valor de normalidad $\left(25 \mathrm{~kg} / \mathrm{m}^{2}\right)$, que fue de $23,0 \pm 1,5 \mathrm{~kg} / \mathrm{m}^{2}$. El restante $44 \%$ de los árbitros, presentaban un IMC por encima de la normalidad que fue de $27,0 \pm 1,4 \mathrm{~kg} / \mathrm{m}^{2}$, siendo esta diferencia estadísticamente significativa $(\mathrm{p}=0,0001)$. Por lo tanto, ningún árbitro uruguayo presentó IMC por encima de la normalidad. Cuando se hizo la comparación de los árbitros brasileños de menor IMC con los árbitros uruguayos, la diferencia estadística también fue significativa $(\mathrm{p}=0,0028)$.

Tabla I. Características antropométricas de los árbitros brasileños y uruguayos.

\begin{tabular}{lrr}
\hline Variables & Brasileños & Uruguayos \\
\hline Edad (años) & $37,6 \pm 4,2$ & $37,8 \pm 2,9$ \\
Masa Corporal $(\mathrm{kg}) *$ & $79,1 \pm 7,9$ & $74,3 \pm 7,4$ \\
Estatura (cm) & $179,1 \pm 4,8$ & $175,5 \pm 6,8$ \\
IMC (kg/m $\left.{ }^{2}\right) *$ & $24,8 \pm 2,4$ & $21,1 \pm 1,6$ \\
Grasa relativa $(\%) *$ & $18,7 \pm 4,4$ & $14,0 \pm 2,4$ \\
Masa muscular $(\%)$ & $42,4 \pm 2,4$ & $42,6 \pm 2,3$ \\
\hline
\end{tabular}

* difiere estadísticamente ( $<<0,05)$. IMC: índice de masa corporal.

En cuanto a las características somatotipológicas de los árbitros brasileños y uruguayos, los resultados pueden ser observados en la Tabla II. La media indicó que los árbitros brasileños y uruguayos evaluados fueron ambos clasificados mediante este método como mesomorfo-endomorfo, ya que registraron un valor promedio de los componentes somatotipológicos de 3,7-4,0-2,0.

Tabla II. Componentes somatotipológicos de los árbitros brasileños y uruguayos.

\begin{tabular}{lcc}
\hline Variables & Brasileños & Uruguayos \\
\hline Endomorfo & $3,7 \pm 1,2$ & $3,7 \pm 1,1$ \\
Mesomorfo & $4,0 \pm 1,4$ & $4,0 \pm 0,8$ \\
Ectomorfo & $2,0 \pm 1,1$ & $2,0 \pm 0,8$ \\
\hline
\end{tabular}

Hubo una heterogenidad considerable entre los datos somatotipológico de los dos grupos (Figs. 1 y 2). Con relación a los árbitros brasileños se puede observar en la somatocarta (Fig. 1) que 10 árbitros presentaron predominancia del componente muscular y grasa sobre el componente estatura (mesomorfo endomorfo), nueve árbitros presentaron predominancia de la grasa y masa muscular sobre el componente estatura (endomorfo mesomorfo), cuatro árbitros con predominancia del componente muscular y estatura sobre la grasa (mesomorfo ectomorfo), tres árbitros con predominancia del componente grasa y estatura sobre la musculatura (endomorfo ectomorfo), culminando con un árbitro que presentó predominancia de la estatura y masa muscular sobre el tejido adiposo (ectomorfo mesomorfo) Figura 1. En la somatocarta de los árbitros uruguayos se pueden observar también datos agrupados, donde cuatro árbitros presentaron predominancia del componente muscular y la grasa sobre la estatura (mesomorfo endomorfo), tres árbitros con predominancia de la grasa y músculo sobre la estatura (endomorfo mesomorfo), dos árbitros con predominancia del componente muscular y estatura sobre la grasa (mesomorfo ectomorfo), un árbitro con predominancia del componente grasa y estatura sobre la musculatura, tres árbitros con equilibrio entre los componentes grasa y muscular (central) y un árbitro con predominancia del componente grasa y estatura sobre el componente muscular (endomorfo ectomorfo) (Fig. 2).

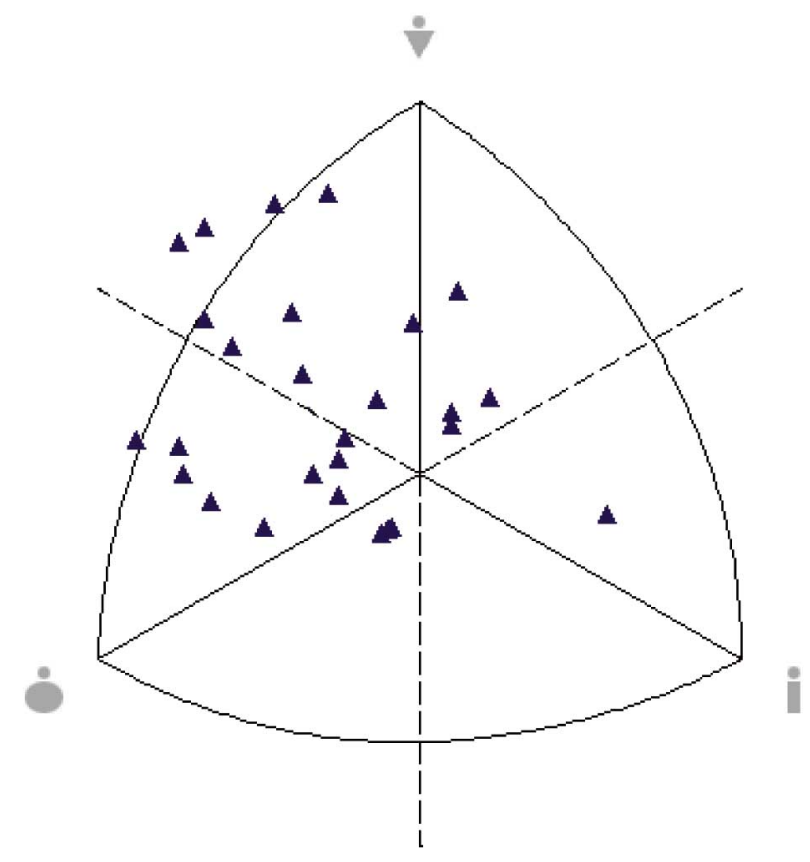

La Fig. 1 exhibe una somatocarta con la distribución de somatopuntos registrados por los árbitros brasileños. 


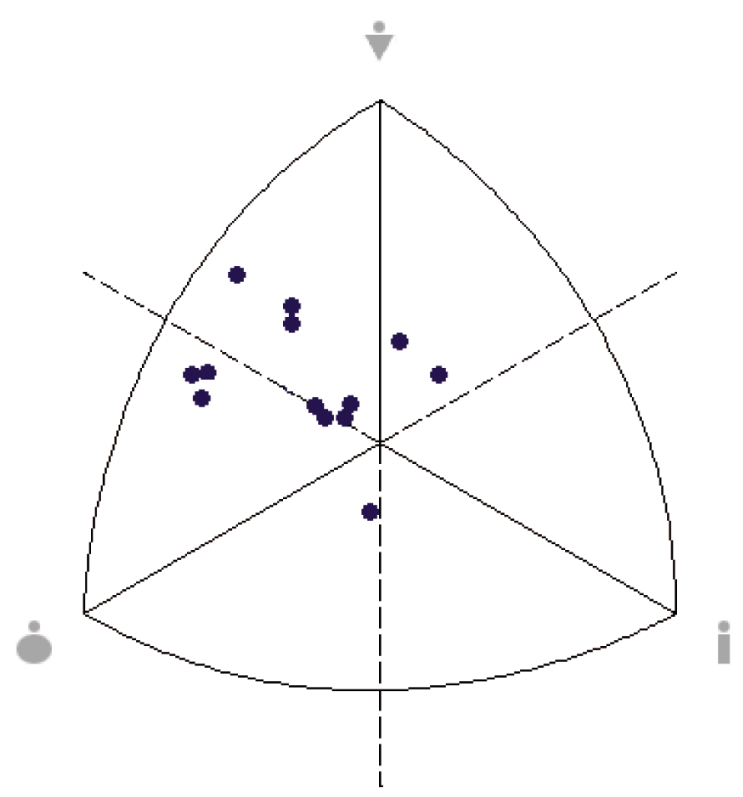

La Fig. 2 exhibe una somatocarta con la distribución de somatopuntos registrados por los árbitros uruguayos.

Sin embargo, la diferencia entre el somatotipo de los dos grupos analizados por la distancia de dispersión del somatotipo (DDS) fue de 0,25 . DDS permite comprobar la distancia entre dos somatotipos al establecer que la diferencia es estadísticamente significativa cuando el valor del DDS es superior a dos. Lo que no se ha observado en los datos de este estudio, es decir, a pesar de la variabilidad individual los grupos no diferían en relación con el somatotipo.

\section{DISCUSIÓN}

La edad promedio de los árbitros de este estudio (Tabla I), no presentaron diferencia estadísticamente significativa, siendo inclusive semejante la edad de árbitros involucrados en otros estudios, como los árbitros griegos 36,3 $\pm 4,5$ años, (Rontoyannis et al.), de árbitros italianos 37,6 \pm 3,4 años (Castagna \& D'Ottavio 2001) de árbitros portugueses $37 \pm$ 6,6 años (Rebelo et al., 2002) de árbitros brasileños $38,9 \pm 38$ (Da Silva et al., 2008) y de los árbitros ingleses $40 \pm 5.1$ (Weston et al., 2009). Algunos estudios afirman que los árbitros de fútbol son en promedio, de 10 a 15 años más viejos que los jugadores de elite de ese deporte, en donde la edad tendría un efecto negativo en el estado físico (Weston et al., 2004). Así siendo así, los árbitros intervenientes en este estudio cumplían esta regla.

La importancia de la diferencia de edad entre los jugadores y árbitros puede estar justificada por la experiencia. Esto es considerado por los órganos rectores del arbitraje internacional de la FIFA y la Union European of Football Association (UEFA) como uno de los requisitos esenciales del individuo para ingresar a la elite del arbitraje (Eissmann \& D'hooghe, 1996). Los estudios demuestran que los árbitros de fútbol para poder arbitrar partidos en la primera línea de nivel nacional e internacional, necesita tener varios años de experiencia (Jones et al., 2002), por lo que los jueces estudiados tenían una edad promedio más de 37 y más de 10 años de experiencia.

Cuando se hace el análisis estadístico de los datos involucrados de los árbitros brasileños y uruguayos referentes a porcentaje de grasa corporal (TablaI) se observa que hay diferencia significativa ( $\mathrm{p}=0,0006)$. Como no hubo diferencia en relación a variable \% de grasa corporal (Tabla I), se infiere que los árbitros brasileños tienen más tejido adiposo, o sea, tienen una carga más para llevar durante un partido. Además de las variables metabólicas, la aptitud física depende de la composición corporal adecuada, ya que exceso de peso u obesidad limita los movimientos además de servir de sobrecarga para el sistema locomotor (Rodriguez-Añez \& Petroski, 2002).

En el caso de los árbitros de fútbol, la función requiere un alto nivel de la demanda metabólica para ser capaces de soportar el clima y la intensidad del partido, lo que hace necesario que presente niveles adecuados de composición corporal y aptitud física, ya que, el árbitro durante un partido de fútbol tiene un gasto de energía media de 740,42 kcal, mientras que el árbitro asistente presenta un gasto promedio de 494,64 kcal (Da Silva \& Rodriguez-Añez, 2001). Según Da Silva et al. el gasto calórico del árbitro en el transcurso del juego es similar a un jugador de fútbol, podría llegar a realizar acciones motoras que corresponden a una intensidad equivalente a 18,4 MET.

En un estudio reciente donde se comparó la composición corporal de árbitros brasileños con árbitros chilenos, se verificó que los árbitros brasileños también presentaban un índice porcentual de grasa superior al de los árbitros chilenos $(19,4 \pm 5,7 \%$ versus 16,6 $\pm 3,5)$ (Bueno et al., 2010). El porcentaje de grasa de los árbitros brasileños de aquel estudio fue parecido al de este trabajo, siendo los árbitros chilenos análogos al de los árbitros uruguayos. Aunque el porcentual de grasa promedio de los árbitros involucrados en este estudio (Tabla I) se encuentre alto, están dentro de la recomendación para hombres que es de 10 a $20 \%$, como objetivo ideal para la salud y buena condición física (Lohman, 1982).

El porcentaje de grasa presentado por árbitros de otra región de Brasil, São Paulo, con edad promedio de 26,75 $\pm 4,13$

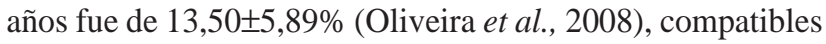
con el porcentaje de grasa corporal presentado por árbitros de elite de España $(11,3 \pm 2,15 \%)$, menor porcentaje encontrado 
por nosotros en la literatura científica (Casajus \& Castagna, 2006). En una investigación que fue llevada a cabo con atletas de fútbol brasileño realizada por Guerra et al. (2004) se observó, por medio del método antropométrico, un valor de adiposidad de $10,6 \%$, siendo que valor semejante $(10,6 \pm 2,6 \%)$ fue encontrado en jugadores de países de América del Sur (Rienzi et al., 1998). Esos valores son inferiores a los presentados por gran parte de los árbitros profesionales de alto nivel que son de mayor edad. Eso demuestra que, con relación al acúmulo de adiposidad, los árbitros evaluados están en condiciones físicas inferiores al compararlos con atletas de esta modalidad. Aunque los árbitros durante el partido están sometidos al mismo gasto calórico del jugador con desplazamientos muy similiares (Da Silva et al.), su biotipo posee características diferentes, principalmente respecto al porcentaje de grasa.

Hubo una heterogeneidad considerable entre los datos del somatotipo de los dos grupos (Figs. 1 y 2), sin embargo, la diferencia entre el somatotipo entre los grupos analizados por la distancia de dispersión del somatotipo (DDS) fue de 0,25. DDS permite comprobar la distancia entre dos somatotipos al establecer que la diferencia es estadísticamente significativa cuando el DDS es 2 (Hebbelinck et al.). Lo que no se ha observado en los datos de este estudio, a pesar de la variabilidad individual grupos no diferían en relación con el somatotipo. Los árbitros fueron clasificados de acuerdo con Carter (2002) como mesomorfo-endomorfo, no hay una superioridad del componente muscular sobre la adiposidad. En una investigación involucrando árbitros de tres categorías, Da Silva \& Rodriguez-Añez (2008) observaron que árbitros más jóvenes (edad promedio de 24,9 \pm 4,6 años) tenían el componente mesomórfico (masa muscular) superior a los árbitros de más edad. Por otra parte, en un estudio longitudinal, (periodo de 10 años), Fidelix y Da Silva (2009) verificaron que con el pasar de los años los individuos que permanecen actuando como árbitro de fútbol van aumentando el porcentaje de grasa corporal.

Estos datos y la literatura científica demuestran que, con respecto a la acumulación de grasa, los árbitros con más experiencia están con déficit físico en comparación con los atletas de fútbol. Con el objetivo de superar este problema, la UEFA y la FIFA han tratado de profesionalizar a sus árbitros. Pues el mundo del fútbol se ha vuelto más competitivo y más rápido en los últimos años, y el árbitro no puede permanecer al margen de este desarrollo (Weston et al.). Además de una buena preparación física con el fin de evaluar las jugadas, evitando que las normas sean violadas, el árbitro también debe estar bien posicionado para ver la agresión entre los deportistas porque el riesgo de que un jugador sufra una lesión es cerca de 1000 veces superior al que se encuentran en la mayoría de otras profesiones (Fuller et al., 2004).

DA SILVA, A. I.; DE LOS SANTOS, H. \& CABRERA, C. Comparative analysis of body composition of football (soccer) referees from Brazil and Uruguay. Int. J. Morphol., 30(3):877-882, 2012.

ABSTRACT: The aim of this study was to comparative analysis of morphological profile of elite soccer referees from Brazil and Uruguay. The sample consisted of 27 referees from Brazil, with a mean age of $37.6 \pm 4.2$ years, weight $79.1 \pm 7.9 \mathrm{~kg}$ and height $179.1 \pm 4.8$ $\mathrm{cm}$; and 14 referees from Uruguay mean age $37.8 \pm 3$ years, weight $74.3 \pm 7.4 \mathrm{~kg}$ and height $175 \pm 6.8 \mathrm{~cm}$. The variables of body weight, height, skinfolds, body girths and bone widths were measured in order to estimate body composition and somatotype of the referees. To determine the body composition we used seven skinfolds, four circumferences and seven widths. With respect to the data of body composition, when comparing the data with the Brazilian and Uruguay, there was a significant statistical difference between body weight, BMI and body fat percentage ( $>$ > 0.05). Although the somatotype of both groups are classified as endomorph-mesomorph (somatotype average 3.7 to 4.0 2.0), the Brazilian officials showed a body fat percentage higher, which might constitute a physical performance limiting factor during the match and could be monitored through a program of good eating habits and physical fitness.

KEY WORDS: Referees; Football (soccer); Body composition.

\section{REFERENCIAS BIBLIOGRÁFICAS}

Bizzini, M.; Junge, A.; Bahr. R. \& Dvorak. J. Injuries and musculoskeletal complaints in referees - A complete survey in the top divisions of the Swiss Football league. Clin J. Sport Medicine, 19:95-100, 2009.

Bouchard, C. Atividade Física e Obesidade. São Paulo, Manole, 2003.

Bueno, J. M.; Da Silva, A. I. \& Fernández, G. E.V. Perfil morfológico de árbitros de futebol do Brasil e do Chile. Rev. Bras. Futebol., 3(2): 28-37, 2010.
Callaway, C. W.; Chumlea, W. C.; Bouchard, C.; Himes, J. H.; Martin, A. D.; Mitchell, C. D.; Mueller, W. H.; Roche, A. F. \& Seefeldt, V. D. Circumferences. In: Lohman, T. G.; Roche, A. F. \& Martorell, R. Anthropometric standardization reference manual. Abridged edition. Human Kinetics Books. Champaign, Illinois, 1991.

Carter, J. E. L. The heath-carter anthropometric somatotype instruction manual. San Diego, USA, 2002. 
Carter, J. E. L. \& Heath, B.H. Somatotyping-Development and Applications. Cambridge University Press, New York, 1990.

Casajus, J. A. \& Castagna, C. Aerobic fitness and field test performance in elite Spanish soccer referees of different ages. J. Sci. Med. Sport, 10(6):382-9, 2007.

Castagna, C. \& D'ottavio, S. Effect of maximal aerobic power on match performance in elite soccer referees. J. Srength and Cond. Res., 15 (4):420-5, 2001.

Cuchiaro, A. L. Relação entre consumo/demanda energética, gordura corporal e estresse. Revista Kinesis, Santa Maria, 22:113-24, 2000.

Dantas, E. H. M. A prática da preparação física. $5^{\mathrm{a}}$ ed. Rio de Janeiro, Shape, 2003.

Da Silva, A. I.; Fernandes, L. C.; Fernandez, R. Energy expenditure and intensity of physical activity in soccer referees during matchplay. J. of Sports Science and Medicine, 7:327-34, 2008.

Da Silva, A. I.; Rodriguez-Añez, C. R. Dispêndio energético do árbitro de do árbitro assistente de futebol. Rev. Educação Físical UEM, 12(2): 113-8, 2001.

Da Silva, A. I.; Rodriguez-Añez, C. R. Somatotipo e composição corporal de árbitros e alunos árbitros de futebol. Rev. Bras Futebol., l(1):20-32, 2008

Eissmann, H. J. \& D'hooghe, M. Sports medical examinations. In: The 23rd Man: Sports Medical Advice for Football Referees. H. J. Eissmann (ed.), Leipzig: Gersoñe-Druck, 1996. pp.7-19.

Fidelix, Y. L. \& Da Silva, A. I. Morfologia do árbitro do futebol após 10 anos na arbitragem. Arq. Ciências Saúde UNIPAR, 14(1):27$35,2010$.

Fuke, K.; Pupo, J. D.; Matheus, C. S. Evaluación de la composición corporal y de la flexibilidad en futbolistas profesionales en diferentes etapas del ciclo de entrenamiento. Archivos de Medicina del Deporte, 26(129):7-13, 2009.

Fuller, C. W.; Junge, A.; Dvorak, J. An assessment of football referees'decisions in incidents leading to player injuries. Am. $J$. Sports Med., 32(1) suppl.: 17s-21s, 2004.

Guedes, D. P. \& Guedes, J. E. R. P. Manual Prático para Avaliação em Educação Física. Barueri, SP: Manole, 2006.

Guerra, I.; Chaves, R.; Barros, T. \& Tirapegui, J. The influence of fluid ingestion on performance of soccer players during a match. J. Sports Science and Medicine, 3:198-202, 2004.

Hebbelinck M, Carter L, \& De Garay A. Body build and somatotype of Olimpic swimmers, divers and water polo players. In: Lewillie L, Clarys JP. Swimming. University Park Press, Baltimore. 285305,1975 .

Jackson, A. S. \& Pollock, M. L. Generalized equations for prediting body density of men. Br. J. Nutr. (40):497-504, 1978.
Jones, M. V.; Paull, G. C. \& Erskine, J. The impact of a team's aggressive reputation on the decision of association football referees. J. Sports Sciences, 20: 991-1000, 2002.

Lohman, T. G. Body composition methodology in sports medicine. The physician and sports medicine, 10(12): 47-48, 1982.

Lohman, T.G.; Roche, A.F. \& Martorell, R. Anthropometric standardization reference manual. Abridged Edition. Human Kinetics Books. Champaign, Illinois, 1991.

Oliveira, M.; Santana, C. H. G. \& Neto, T. L. B. Análise dos padrões de movimento e dos índices funcionais de árbitros durante uma partida de futebol. Fitness \& Performance Journal, 7(1):41-47, 2008.

Reilly, T. \& Gregson, W. Special populations: The referee and assistant referee. J. Sports Sci., 24(7): 795-801, 2006.

Rebelo, A.; Silva, S.; Pereira, N. \& Soares, J. Stress físico do árbitro de futebol no jogo. Rev. Portuguesa de Ciências do Desporte, 2(5): 24-30, 2002

Rienzi, E.; Mazza. J. C.; Carter, J. E. L. \& Reilly, T. Futbolista Sudamericano de Elite: Morfología, Análisis del Juego y Performance. Rosario, Biosystem Servicio Educativo, 1998.

Rodriguez-Añez, C. R. \& Petroski, E. L. O exercício físico no controle do sobrepeso e da obesidade. Lecturas Educación Fisica y Deportes [periódico na Internet]. 2002 Septiembre [cited 2009 abril 23] 8(52): [cerda de 5 p.] Disponível em: http:// www.efdeportes.com/efd52/obesid.htm

Rontoyannis, G.P.; Stalikas, A.; Sarros, G. \& Vlastaris, A. Medical, morphological and funcional aspects of Greek football referees. J. of Sports Medicine and Physical Fitness, (38):208-14, 1998.

Siri, W. E. Body composition from fluid space and density. In: Brozek, J. Hanschel, A. Techniques for measuring body composition. Washington, D.C. National Academy of Science, p.223-224, 1961.

Weston, M.; Helsen, W.; Macmahon, C. \& Kirkendall, D. The impact of specific high-intensity training sessions on football referees' fitness levels. Am. J. of Sports Medicine, 32(1) suppl.: 54s-61s, 2004.

Weston, M.; Catagna, C.; Helson, W. \& Impellizzeri, F. M. Relationships among field-test measures and physical match performance in elite-standard soccer referees J. Sports Sciences, 27(11):1177-84, 2009.

Correspondence to:

Alberto Inácio da Silva

Docente da Universidade Estadual de Ponta Grossa -

Paraná

BRASIL

Email: albertoinacio@bol.com.br

Received: 02-03-2012

Accepted: 29-06-2012 